\title{
FINITE DIFFERENCE METHOD IMPLEMENTATION FOR NUMERICALINTEGRATION HYDRODYNAMIC EQUATIONS MELTS
}

\author{
Kazhikenova S.Sh. ${ }^{1}$, Belomestny D. ${ }^{2}$, Shaltakov S.N. ${ }^{1}$, Shaihova G.S. ${ }^{1}$ \\ ${ }^{1}$ Karaganda State Technical University, Karaganda, Kazakhstan, sauleshka555@mail.ru \\ 2Duisburg-Essen University, Duisburg, Germany
}

\begin{abstract}
The liquid state theory is not a simple section of the modern theory of metallurgical processes. Any substance in liquid state is a difficult object to establish not only quantitative, but also qualitative patterns, being that liquid state is intermediate between solid and gaseous states. Theoretical hydrodynamics has long attracted attention of various specialties' scientists: comparative simplicity of the basic equations, precise problems formulation and clarity of its experiments inspired hope of getting a dynamic phenomena's complete description occurring in melts. In describing continuous media's dynamic properties the following systems of equations were obtained: for a viscous melt - the Navier Stokes equations, for an ideal melt - the Euler equations, for a weakly compressible melt - the Oberbeck - Boussinesq equations. In fundamental research and in the field of applied research these mathematical models are generally accepted for modeling melt flow. Theoretical processes descriptions occurring in melts are based on the Stokes - Kirchhoff theory, which, with the frame of classical hydrodynamics, revealed phenomenological connections between the molten systems' kinetic properties. Numerous hydrodynamic paradoxes point to that long and thorny path that has been covered since its inception. First long stage was associated with the study and research of ideal incompressible liquid's potential flows. Mathematical methods of their research using the theory of complex variable functions seemed almost perfect. Imperfection of the ideal liquid theory was indicated by the famous Euler-Dalamber paradox: the total force acting on a body flowing around a potential flow is equal to zero. Then a mathematical model of a viscous incompressible fluid with its basic Navier-Stokes equations was created. Proposed section outlines various methods for solving and studying the Navier - Stokes equations. At the present stage, a great effort is made to find localized hydrodynamics equations solutions.
\end{abstract}

Keywords: Metal melt, hydrodynamic equations, velocity profile, mathematical modeling, computer simulation, density functional

\section{Introduction}

Objective - to obtain the most simple regularization of the original system of hydrodynamic equations containing a physical sense. As known [1,2], hydrodynamic equations approximation leads to non-linear systems of equations. Therefore, their solution is accompanied by complex problems. These problems create difficulties in solving multidimensional tasks using fairly wellknown implicit schemes in time. The task of obtaining original system's the simplest regularization, containing a certain physical meaning, becomes urgent. In order to solve these tasks, in our opinion, most constructive approach is a splitting method. In this connection, we considered various approaches to the splitting schemes construction for the Navier - Stokes equations in the weak approximation sense.

Main task of article is to determine the viscous incompressible melt's motion, if external forces acting on the melt are known, the boundary mode and, for a non-stationary flow, the initial velocity field. Basically, we assume that there is a coordinate system in which the filled with the melt is unchanged. The assumption of the field's constancy is fulfilled in such practically important tasks as the problem of flowing a solid body with an infinite flow; the problem of the liquid motion under 
the action of volume forces in a vessel with solid walls moving in a known manner in space and others.

\section{Decision problem}

We consider a flat flow. Let $\Omega$-area of Euclidean space $R^{n}$, and $x=\left(x_{1}, x_{2}\right)$. We divide whole space $R^{n}(x, t)$ on elementary cells, the area of which:

$$
x_{i}=k_{i} h, \quad h>0, \quad k_{i}=0, \pm 1, \pm 2 \ldots
$$

where $t=k \Delta t ; k=1,2, \ldots, n ; h-$ step.

We form difference ratiosby $x_{i}$ :

$$
v_{x_{i}}(x, t)=\frac{1}{h}\left[v\left(x+h e^{j}, t\right)-v(x, t)\right], \quad v_{\bar{x}_{i}}(x, t)=\frac{1}{h}\left[v(x, t)-v\left(x-h e^{j}, t\right)\right]
$$

Shift by $x_{i}$ is defined as:

$$
\stackrel{ \pm i}{v(x, t)}=v\left(x \pm h e^{j}, t\right) .
$$

Consider the temperature model of inhomogeneous melt $[3,4]$ in the area $\Omega \subset R^{2}$ :

$$
\begin{aligned}
& \rho\left(\frac{\partial v}{\partial t}+(v \cdot \nabla) v\right)=\mu \Delta v-\nabla p+e \theta \rho+\rho f, \\
& \frac{\partial \rho}{\partial t}+(v \cdot \nabla) \rho=0 \\
& \operatorname{div} v=0 \\
& \rho\left(\frac{\partial \theta}{\partial t}+(v \cdot \nabla) \theta\right)=\operatorname{div}(\lambda(\theta) \nabla \theta)+\mu \sigma, \\
& \sigma=\sum_{i, j=1}^{2}\left(\frac{\partial v_{i}}{\partial x_{j}}+\frac{\partial v_{j}}{\partial x_{i}}\right)^{2},
\end{aligned}
$$

with initial boundary conditions:

$$
\left.v\right|_{t=0}=v_{0}(x),\left.\rho\right|_{t=0}=\rho_{0}(x),\left.\theta\right|_{t=0}=\theta_{0}(x),\left.v\right|_{S}=0,\left.\frac{\partial \theta}{\partial n}\right|_{S}=0, t \in[0, T],
$$

where $\sigma$-is energy dissipation, $v(x, t)$ - velocities' vector function, $\theta(x, t)$-temperature field, $\rho(x, t)$-density field, $p(x, t)$-pressure field, $f(x, t)$-mass force vector, $\mu$-melt viscosity, $\lambda(\theta)$-thermal conductivity coefficient, $n$ - external normal to the boundary of $S, e=\{0,1\}$.

In order to demonstrate given method after appropriate transformations, we rewrite equation (1) in form:

$$
\frac{\partial v}{\partial t}+\sum_{k=1}^{n} Z_{k}(v)-\frac{1}{\varepsilon} \nabla d i v v=f,
$$

where:

$$
Z_{k}(w)=-\gamma \frac{\partial^{2} w}{\partial x_{k}^{2}}+v_{k} \frac{\partial w}{\partial x_{k}}+\frac{1}{2} \frac{\partial v_{k}}{\partial x_{k}} w
$$


There are various approximations of difference operator $Z_{k}$. We take this operator in the form proposed in [4-9]:

$$
Z_{k}^{m}(w)=-2 w_{x_{k} \bar{x}_{k}}+\frac{1}{2} v_{k}^{+k^{m-\frac{k}{2}}} w_{x_{k}}+\frac{1}{2} v_{k}^{m-\frac{k}{2}} w_{\bar{x}_{k}}+\frac{1}{2} v_{k x_{k}}^{m-\frac{k}{2}} w_{x_{k}}
$$

Then equation (2) can be represented by following difference scheme:

$$
\begin{aligned}
& \frac{1}{\Delta t}\left(v_{1}^{m-\frac{1}{2}}-v_{1}^{m-1}\right)+\tau_{2}^{m}\left(v_{1}^{m-\frac{1}{2}}\right)=\frac{1}{2} f_{1}^{m-\frac{1}{2},} \\
& \frac{1}{\Delta t}\left(v_{1}^{m-\frac{1}{2}}-v_{1}^{m-1}\right)+\tau_{2}^{m}\left(v_{1}^{m-\frac{1}{2}}\right)=\frac{1}{2} f_{1}^{m-\frac{1}{2}}, \\
& \frac{1}{\Delta t}\left(v_{1}^{m}-v_{1}^{m-\frac{1}{2}}\right)+\tau_{1}^{m}\left(v_{1}^{m}\right)-\frac{1}{\varepsilon}\left(v_{1 x_{1}}^{m}+v_{2 x_{2}}^{m-\frac{1}{2}}\right)_{\bar{x}_{1}}=\frac{1}{2} f_{1}^{m}, \\
& \frac{1}{\Delta t}\left(v_{2}^{m-\frac{1}{2}}-v_{2}^{m}\right)+\tau_{2}^{m}\left(v_{2}^{m-\frac{1}{2}}\right)-\frac{1}{\varepsilon}\left(v_{1 x_{1}}^{m-1}+v_{2 x_{2}}^{m-\frac{1}{2}}\right)_{\bar{x}_{2}}=\frac{1}{2} f_{2}^{m-\frac{1}{2}}, \\
& \frac{1}{\Delta t}\left(v_{2}^{m}-v_{2}^{m-\frac{1}{2}}\right)+\tau_{1}^{m}\left(v_{2}^{m}\right)=\frac{1}{2} f_{2}^{m},
\end{aligned}
$$

where $m=1,2, \ldots, N$.

To complete construction of the difference scheme, initial and boundary conditions should be added to these equations. Without deriving formulas for boundary conditions, we write:

$$
\begin{aligned}
& \left\|v_{1}^{m-\frac{1}{2}}\right\|^{2}-\left\|v_{1}^{m-1}\right\|^{2}+\left\|v_{1}^{m-\frac{1}{2}}-v_{1}^{m-1}\right\|^{2}+2 \gamma \Delta t\left\|v_{1 x_{2}}^{m-\frac{1}{2}}\right\|^{2}=\Delta t\left(f_{1}^{m-\frac{1}{2}}, v_{1}^{m-\frac{1}{2}}\right) \text {, } \\
& \left\|v_{1}^{m}\right\|^{2}-\left\|v_{1}^{m-\frac{1}{2}}\right\|^{2}+\left\|v_{1}^{m}-v_{1}^{m-\frac{1}{2}}\right\|^{2}+2 \gamma \Delta t\left\|v_{1 x_{1}}^{m}\right\|^{2}+ \\
& +\frac{2 \Delta t}{\varepsilon}\left[\left\|v_{1 x_{1}}^{m}\right\|^{2}+\left(v_{1 x_{1}}^{m}, v_{2 x_{2}}^{m-\frac{1}{2}}\right)\right]=\Delta t\left(f_{1}^{m}, v_{1}^{m}\right), \\
& \left\|v_{2}^{m-\frac{1}{2}}\right\|^{2}-\left\|v_{2}^{m-1}\right\|^{2}+\left\|v_{2}^{m-\frac{1}{2}}-v_{2}^{m-1}\right\|^{2}+2 \gamma \Delta t\left\|\begin{array}{c}
m-\frac{1}{2} \\
v_{2 x_{2}}^{2}
\end{array}\right\|^{2}+ \\
& +\frac{2 \Delta t}{\varepsilon}\left[\left\|v_{2 x_{2}}^{m-\frac{1}{2}}\right\|^{2}+\left(v_{1 x_{1}}^{m-1}, v_{2 x_{2}}^{m-\frac{1}{2}}\right)\right]=\Delta t\left(f_{2}^{m-\frac{1}{2}}, v_{2}^{m-\frac{1}{2}}\right) \\
& \left\|v_{2}^{m}\right\|^{2}-\left\|v_{2}^{m-\frac{1}{2}}\right\|^{2}+\left\|v_{2}^{m}-v_{2}^{m-\frac{1}{2}}\right\|^{2}+2 \gamma \Delta t\left\|v_{2 x_{1}}^{m}\right\|^{2}=\Delta t\left(f_{2}^{m}, v_{2}^{m}\right) .
\end{aligned}
$$


Thus, we obtain equations (3) - (6), which are solved separately. This allows you to write machine programs for the numerical finite-difference methods implementation. We consider application of proposed method on Dirichlet problem example for the Poisson equation given in [615]. Integration is performed in a rectangular lattice in accordance with fig. 1. Asterisk indicates internal nodes, boundary nodes are denoted by «"»».

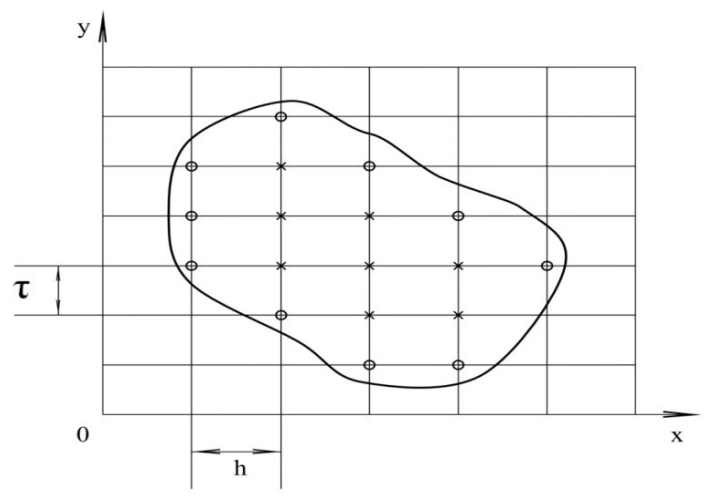

Fig.1. Integration area

\section{Results and discussions}

According to the reference data, solution of the Poisson equation is given in table 1. For the control example in Table 2 we give the Dirichlet problem's solution already with different boundary conditions from same reference sources. Comparing first and second Dirichlet boundary value problems' solutions from reference sources presented in tables 1 and 2 with program results for solving boundary value problems presented in tables 3 and 4, we see a satisfactory coincidence of solutions for a given accuracy $\varepsilon=10^{-1}$.

Table 1. First Dirichlet boundary value problem's solution for the Poisson equation from reference sources

\begin{tabular}{|c|c|c|c|c|c|c|}
\hline \multirow{2}{*}{$\mathrm{Y}$} & \multicolumn{7}{|c|}{$\mathrm{X}$} \\
\cline { 2 - 7 } & 0.00 & 0.40 & 0.80 & 1.20 & 1.60 & 2.00 \\
\hline 0.00 & 0.00 & 0.00 & 0.00 & 0.00 & 0.00 & 0.00 \\
\hline 0.20 & 0.08 & 0.32 & 0.51 & 0.72 & 0.99 & 0.84 \\
\hline 0.40 & 0.32 & 0.72 & 1.07 & 1.41 & 1.78 & 1.76 \\
\hline 0.60 & 0.72 & 1.23 & 1.68 & 2.12 & 2.56 & 2.76 \\
\hline 0.80 & 1.28 & 1.82 & 2.65 & 3.22 & 3.82 & 3.84 \\
\hline 1.00 & 2.00 & 2.44 & 2.96 & 3.56 & 4.24 & 5.00 \\
\hline
\end{tabular}

Table 2. Second Dirichlet boundary value problem's solution for Poisson equation from reference sources

\begin{tabular}{|c|c|c|c|c|c|c|}
\hline \multirow{2}{*}{$\mathrm{Y}$} & \multicolumn{7}{|c|}{$\mathrm{X}$} \\
\cline { 2 - 7 } & 0.00 & 0.40 & 0.80 & 1.20 & 1.60 & 2.00 \\
\hline 0.00 & 1.00 & 1.40 & 1.80 & 2.20 & 2.60 & 3.00 \\
\hline 0.20 & 2.00 & 1.05 & 0.95 & 1.08 & 1.44 & 2.96 \\
\hline 0.40 & 2.00 & 1.02 & 0.60 & 0.59 & 0.93 & 2.84 \\
\hline 0.60 & 4.00 & 1.36 & 0.78 & 0.63 & 0.93 & 2.64 \\
\hline 0.80 & 5.00 & 2.78 & 2.12 & 1.81 & 1.64 & 2.36 \\
\hline 1.00 & 6.00 & 5.84 & 5.36 & 4.56 & 3.44 & 2.00 \\
\hline
\end{tabular}


Engineering: Modeling of the nonlinear physical and technical processes. 149

Table 3 - First Dirichlet boundary value problem's solution for the Poisson equation with a given accuracy $\varepsilon=10^{-1}$

\begin{tabular}{|c|l|l|l|l|l|l|l|l|l|l|l|}
\hline $\mathrm{Y}$ & \multicolumn{10}{|c|}{$\mathrm{X}$} \\
\cline { 2 - 15 } & 0.000 & 0.200 & 0.400 & 0.600 & 0.800 & 1.000 & 1.200 & 1.400 & 1.600 & 1.800 & 2.000 \\
\hline 0.0 & 0.000 & 0.000 & 0.000 & 0.000 & 0.000 & 0.000 & 0.000 & 0.000 & 0.000 & 0.000 & 0.000 \\
\hline 0.2 & 0.080 & 0.241 & 0.262 & 0.264 & 0.266 & 0.269 & 0.272 & 0.276 & 0.280 & 0.330 & 0.840 \\
\hline 0.4 & 0.320 & 0.303 & 0.301 & 0.303 & 0.305 & 0.308 & 0.311 & 0.315 & 0.320 & 0.447 & 1.760 \\
\hline 0.6 & 0.720 & 0.356 & 0.305 & 0.306 & 0.308 & 0.310 & 0.314 & 0.318 & 0.323 & 0.538 & 2.760 \\
\hline 0.8 & 1.280 & 0.429 & 0.310 & 0.308 & 0.310 & 0.313 & 0.316 & 0.320 & 0.325 & 0.636 & 3.840 \\
\hline 1.0 & 2.000 & 0.523 & 0.315 & 0.311 & 0.313 & 0.315 & 0.319 & 0.323 & 0.329 & 0.741 & 5.000 \\
\hline 1.2 & 2.880 & 0.639 & 0.322 & 0.314 & 0.316 & 0.319 & 0.322 & 0.326 & 0.332 & 0.854 & 6.240 \\
\hline 1.4 & 3.920 & 0.776 & 0.330 & 0.318 & 0.320 & 0.323 & 0.326 & 0.330 & 0.337 & 0.974 & 7.560 \\
\hline 1.6 & 5.120 & 0.935 & 0.341 & 0.323 & 0.326 & 0.329 & 0.332 & 0.336 & 0.343 & 1.105 & 8.960 \\
\hline 1.8 & 6.480 & 1.248 & 0.581 & 0.613 & 0.674 & 0.744 & 0.821 & 0.906 & 1.002 & 1.946 & 10.44 \\
\hline 2.0 & 2.000 & 2.440 & 2.960 & 3.560 & 4.240 & 5.000 & 5.840 & 6.760 & 7.760 & 8.840 & 10.00 \\
\hline
\end{tabular}

Table 4 - Second Dirichlet boundary value problem's solution for the Poisson equation with a given accuracy $\varepsilon=10^{-1}$

\begin{tabular}{|c|l|l|l|l|l|l|l|l|l|l|l|}
\hline $\mathrm{Y}$ & \multicolumn{10}{|c|}{$\mathrm{X}$} \\
\cline { 2 - 14 } & 0.000 & 0.200 & 0.400 & 0.600 & 0.800 & 1.000 & 1.200 & 1.400 & 1.600 & 1.800 & 2.000 \\
\hline 0.0 & 1.000 & 1.200 & 1.400 & 1.600 & 1.800 & 2.000 & 2.200 & 2.400 & 2.600 & 2.800 & 3.000 \\
\hline 0.2 & 2.000 & 1.109 & 1.011 & 1.012 & 1.016 & 1.019 & 1.023 & 1.026 & 1.029 & 1.138 & 2.960 \\
\hline 0.4 & 3.000 & 1.215 & 1.004 & 0.997 & 0.996 & 0.994 & 0.992 & 0.990 & 0.988 & 1.087 & 2.840 \\
\hline 0.6 & 4.000 & 1.325 & 1.007 & 0.997 & 0.995 & 0.993 & 0.991 & 0.988 & 0.985 & 1.074 & 2.640 \\
\hline 0.8 & 5.000 & 1.436 & 1.009 & 0.996 & 0.994 & 0.992 & 0.989 & 0.986 & 0.983 & 1.057 & 2.360 \\
\hline 1.0 & 6.000 & 1.546 & 1.012 & 0.995 & 0.993 & 0.990 & 0.987 & 0.984 & 0.981 & 1.035 & 2.000 \\
\hline 1.2 & 7.000 & 1.656 & 1.015 & 0.994 & 0.992 & 0.989 & 0.986 & 0.983 & 0.979 & 1.008 & 1.560 \\
\hline 1.4 & 8.000 & 1.767 & 1.017 & 0.994 & 0.991 & 0.988 & 0.984 & 0.981 & 0.977 & 0.977 & 1.040 \\
\hline 1.6 & 9.000 & 1.877 & 1.020 & 0.993 & 0.990 & 0.986 & 0.983 & 0.979 & 0.975 & 0.942 & 0.440 \\
\hline 1.8 & 10.00 & 2.018 & 1.059 & 1.027 & 1.022 & 1.016 & 1.009 & 1.000 & 0.992 & 0.917 & -0.24 \\
\hline 2.0 & 6.000 & 5.960 & 5.840 & 5.640 & 5.360 & 5.000 & 4.560 & 4.040 & 3.440 & 2.760 & 2.000 \\
\hline
\end{tabular}

Table 5 - First Dirichlet boundary value problem's solution for the Poisson equation with a given accuracy $\varepsilon=10^{-4}$

\begin{tabular}{|c|c|c|c|c|c|c|}
\hline \multirow{2}{*}{$\mathrm{Y}$} & \multicolumn{7}{|c|}{$\mathrm{X}$} \\
\cline { 2 - 7 } & 0.000 & 0.400 & 0.800 & 1.200 & 1.600 & 2.000 \\
\hline 0.00 & 0.000 & 0.000 & 0.000 & 0.000 & 0.000 & 0.000 \\
\hline 0.20 & 0.080 & 0.301 & 0.508 & 0.750 & 1.001 & 0.800 \\
\hline 0.40 & 0.320 & 0.730 & 1.055 & 1.430 & 1.851 & 1.710 \\
\hline 0.60 & 0.720 & 1.221 & 1.666 & 2.101 & 2.590 & 2.732 \\
\hline 0.80 & 1.280 & 1.790 & 2.599 & 3.202 & 3.798 & 3.884 \\
\hline 1.00 & 2.000 & 2.490 & 2.981 & 3.549 & 4.290 & 5.001 \\
\hline
\end{tabular}

It should be noted that the increase in accuracy leads to an increase in the cost of machine time, which is 45 minutes. In general, a large number of experiments have been made for increase in accuracy of mathematical model, with a step of the tension of the x-ray radiation of $5 \mathrm{kV}$. 


\section{Conclusion}

Obtained results show compiled program's correctness, as well as correctness of the stated boundary value problems for hydrodynamic equations considered by us above. In this article, we establish one of the important moments of the Navier - Stokes equations' theory: the unique stationary problems' solvability in the case of their linearization. This is most easily done in a Hilbert space with a well-defined extension of the solution concept, which will be described below.Here are specific algorithms for computer programming.

\section{REFERENCES}

1 Conca Carlos. On the application of the homogenization theory to a class of problems arising in fluid mechanics. J. Math.Pursat Appl. 1985, Vol.64, No.1, pp. $31-35$.

2 Malik M.R., Zang T.A., Hussaini M.Y. A spectral collocation method for the Navier-Stokes equations. J. Comput. Phys. 1985, Vol. 61, No.1, pp. $64-68$.

3 Gresho P.M. Incompressible fluid dynamics: some fundamental formulation issues. Annu. Rev. Fluid Mech., 1991, Vol.23, pp. 413 - 453. [in Russian]

4 Issagulov A.Z., Belomestny D., Shaikhova G.S., et al. Functions of atoms radial distribution and pair potential of some semiconductors melts. The Bulletin the National Academy of Sciences of the Republic of Kazakhstan. 2019, No. 4, pp. 6 - 14. DOI: 10.32014/2019.2518-1467.86

5 Kazhikenova S.Sh., Belomestny D. Fundamental Characteristics of Reliability in Technological Processes in Ferrous Metal Industry. The Bulletin the National Academy of Sciences of the Republic of Kazakhstan. 2019, No. 2, pp. 120 - 127. DOI: 10.32014/2019.2518-1467.50

6 Ladyzhenskaya O.A. Mathematical problems of the dynamics of a viscous incompressible fluid. M. Nauka, 1970, 288p. [in Russian]

7 Kazhikenova S.Sh. On the difference method of the ocean model. Bulletin of KazNU. Ser. mat., fur., inf. 2002, No. 3 (31), pp. 99 - 101. [in Russian]

8 Abramov A., Yukhno L.F. Solving some problems for systems of linear ordinary differential equations with redundant conditions. Zh. Vychisl. Mat. Mat. Fiz, 2017, No. 57:8, pp. 1285 - 1293, https://doi.org/10.7868/S00444 66917080026

9 KatoYasumasa, Tanahashi Takahico. Finite-element method for three-dimensional incompressible viscous flow using simultaneous relaxation of velocity and Bernoulli function. 1st report flow in a lid-driven cubic cavity at Re=5000. Trans. Jap. Soc. Mech. Eng. 1991, Vol. 57, No.540, pp. $2640-2647$.

10 Honda Itsuro, Ohba Hideki, Tanigawa Yuji, Nakiama Tetsuji. Numerical analysis of a flow in a three-dimensional cubic cavity. Trans. Jap. Soc. Mech. Eng. 1991, Vol. 57, No. 540, pp. 2627-2631.

11 Yan X. Wei L, Lei Yao, Xin Xue, et al. Numerical Simulation of Meso-Micro Structure in NiBased Superalloy During Liquid Metal Cooling. Proceedings of the 4th World Congress on Integrated Computational Materials Engineering. The Minerals, Metals \& Materials Series. 2017, pp. 249 - 259.

12 Barannyk, T.A., Barannyk A.F., Yuryk I.I. Exact Solutions of the Nonliear Equation. Ukrains'kyi Matematychnyi Zhurnal, 2017, Vol. 69, No. 9, pp. 1180-1186

13 Tleugabulov S., Ryzhonkov D., Aytbayev N., Koishina G., Sultamurat G. The reduction smelting of metal-containing industrial wastes. News of the National Academy of Sciences of the Republic of Kazakhstan, Series of Geology and Technical Sciences, 2019, Vol 1, No. 433, pp. 32 - 37. https://doi.org/10.32014/2019.2518-170X.3

14 Skorokhodov S.L., Kuzmina N.P. Analytical-numerical method for solving an Orr-Sommerfeldtype problem for analysis of instability of ocean currents. Zh. Vychisl. Mat.\& Mat. Fiz., 2018, No. 8:6, pp. 1022-1039; https://doi.org/10.7868/S00444669 18060133

15 Iskakova, N.B., Assanova A.T., Bakirova E.A. Numerical Method for the Solution of linear boundary-Value Problem for Integro-differential Equations Based on spline approximations. Ukrains'kyi Matematychnyi Zhurnal, 2019, Vol. 71, No. 9, pp. 1176 - 1191. http://umj.imath.kiev.ua/index.php/umj /article/view/1508. 